\title{
Conversation analysis, cyberpsychology and online interaction
}

\begin{abstract}
In this paper, I explore how conversation analysis can be used as a method for analysing online interaction. As the number and quantity of online communication platforms have proliferated, there has been a growing interest in social psychology about the impact and effectiveness of online, text-based communication. A number of theories have been used and developed to explain how online communication might impact upon relationships and effective communication. However, this paper argues that in order to explore the differences between online and offline interaction an analysis of online behaviour is needed. Conversation analysis allows for an in-depth, sequential and discursive analysis of real-life online interactions. It can explore the ways in which the affordances of the interactional platforms are oriented to or made relevant in the interaction. The utility of conversation analysis is demonstrated through a number of examples, highlighting how this method can be used to broaden our understanding of how online communication works in practice.
\end{abstract}


For social psychologists, there has been an interest in the role that the internet has played in changing our behaviours. The internet has potentially impacted upon many aspects of our lives, such as the way in which we conduct our relationships (Myddleton \& Attrill, 2015), the construction and presentation of stereotypes (Bailey, Steeves, Burkell \& Raven, 2013), the ways in which we behave in groups (Postmes, Spears \& Lee, 2000), and our pro-social behaviour (Cox, Nguyen, Thorpe, Ishizaka, Chakhar, \& Meech, 2018) amongst many other things. There is also an implicit (and often explicit) interest in the ways in which communication and interaction impact upon our behaviours; for example, how we communicate when developing relationships (Speer, 2017). Since the mid-1990s, much of our communication is now taking place online. For example, there are currently an estimated 269 billion e-mails, 55 billion Whatsapp messages, over 9.5 billion Instagram photos and videos, and around 500 million tweets created per day. For social psychologists there has been a long-term interest in how online and offline interaction might differ (McKenna \& Bargh, 2000) and how this might then impact upon the effectiveness of online communication in comparison to spoken interaction (Walther, 1996). In this paper I will argue that in order to understand online communication we need to analyse how individuals actually interact in online contexts. I will argue that conversation analysis presents an appropriate method for analysing online interaction, and allows us to rigorously compare spoken and online interaction.

\section{Online and offline interaction}

There have been various approaches taken to understanding the differences between online and offline interaction. In the earliest days of the internet, a number of theories were posited which come under the umbrella of 'cues-filtered-out' theories (Culnan \& Markus, 1987; Walther, 2011). These theories very broadly stated that due to the lack of non-verbal cues, such as body language, facial expressions, tone of voice and so on, it is not possible for users to form truly interpersonal connections. Many of these theories were imported from a pre-internet era to make claims about online communication. Media richness theory (Daft \& Lengel, 1986) originally focused on the effectiveness of different communication channels for organizational decision-making. The argument was that when media was less rich, that is, when it had fewer channels of communication, it was less effective in terms of a communication strategy because there was the potential for more equivocality in the message. When this is transposed to online interactions, it suggests that when communication is text-based, and so less rich, there is the higher potential for multiple interpretations of a message and so miscommunication may occur. Another theory proposed that because there were fewer social context cues, users were less attuned to each other and more individuated and as such they were more likely to become disinhibited and belligerent (Sproull \& Kiesler, 1986). Similarly, social presence theory (Short, Williams \& Christie, 1976) argued that the 
fewer cues a communicative platform supported, the less warmth and involvement users experienced with one another. In other words, they were less likely to feel that they were interacting with another human being. These theories all focused on the fact that without access to the social cues that we take for granted in face-to-face interaction, we experience less closeness with the individuals with whom we are interacting. More recent theories, such as online disinhibition (Suler, 2004) suggest that these features can lead to toxic disinhibition which may lead to negative or abusive behaviour online. Of course, it is also important to note that Suler suggested that this may also lead to benign disinhibition which may allow for people to develop stronger relationships online.

There are also more nuanced theories and explanations of online communication which focus on the context of the interaction as well as the platforms through which it takes place. Social information processing theory (SIP; Walther, 1992) builds on the ideas of the reduced cues theories but with some adaptations. SIP presumes that individuals are driven to build social relationships, whether online or offline. However, in an online context the development of these relationships may be slowed down, because of the rate of social exchange. As there are fewer cues, this means that less social information is available about an interlocutor in each message, and as such it takes longer for parties in an interaction to build a relationship. SIP still, then, considers that reduced cues are an issue, but acknowledges that parties can still build a relationship. Walther (1996) further developed these ideas with the hyperpersonal model of computer-mediated communication. This model suggests that there may be processes online which can lead to more intimate relationships developing online than in a parallel offline interaction. The model posits four different features of online communication which may lead to this hyperpersonal effect. The first is that receivers, in the absence of other social context cues, may exaggerate their impression of the sender. The second is that the sender is able to have much more control over the impression that they are making. They can also avoid any impressions being formed on the basis of unconscious aspects, such as facial expressions, mismanaged eye contact, interrupting and so on (Walther, 2011). The third aspect is related to the features of the channel, which allow for users to write and re-write their messages to ensure that they make the best impression when it is eventually sent. The final feature relates to how the first three features combine, which allows for a positive feedback loop to be created. When a sender has spent time creating a 'perfect' message, and the receiver exaggerates their perceptions of that person, then they are more likely to also send a favourable message in return. In this way, users can develop intimate feelings for one another more quickly than they may in face-to-face interaction. This theory has been supported by a number of experimental studies (Walther, 1996) as well as interview studies around online dating (Ellison, Heino \& Gibbs, 2006; Farrer \& Gavin, 2009). 
These theories have an underlying presumption that online interaction is inherently different to spoken interaction, and as such, it needs explaining in some way. However, few of these theories seem to start from a detailed analysis of online behaviour. If we want to understand how individuals behave in online contexts, it would seem to be self-evident that we need to examine actual behaviour as it occurs in real-world situations. Studies of online behaviour and language have been undertaken by those engaged in Computer-Mediated Discourse Analysis (CMDA; Herring, 2004). CMDA draws on a variety of methods and concepts from a variety of different research areas, including conversation analysis (Androutsopoulos \& Beisswenger, 2008). These types of studies have challenged some of the theories around reduced cues and shown how individuals have adapted their communicative practices to the potential constraints of the medium (Herring \& Androutsopoulous, 2015). CMDA comprises a very broad range of methods, which can be both qualitative and quantitative, and can focus on many different research questions (Androutsopoulos \& Beisswenger, 2008). The aim of this paper is to concentrate specifically on conversation analysis (CA) to demonstrate how this method allows us to examine the sequential unfolding of online interaction in context.

\section{Conversation analysis}

Conversation analysis (CA) focuses on the sequential organization of interaction (Schegloff, 2007), and explore social interaction in order to find the normative practices of interaction (Potter, 2012a). CA offers a method to explicate the "processes of social interaction, shared meaning, mutual understanding, and the coordination of human conduct" (Goodwin \& Heritage, 1990, p. 283). The aim of CA is to "examine how, through talking, people live their lives, build and maintain relationships and establish who they are to one another" (Stokoe, 2009, p. 81). It is important to note that CA is not a method or a theory of communication, but rather aims to empirically discover patterns of talk (Antaki, 1994). Its focus is not on "conversation" in and of itself, but rather how that conversation illuminates actions, events and objects (Pomerantz \& Fehr, 1997). It has been used within social psychology to explore a wide variety of areas, such as the recognition of flirting (Speer, 2017), the use of categories in action (Antaki \& Widdicome, 1998), and the social psychology of emotion (Potter \& Hepburn, 2019).

CA makes a number of core assumptions about conversation and its role in social action. CA finds that talk is action-oriented; in other words, it analyses the talk for how it "does things, accomplishes things" (Edwards, 2004, p.43). It is important here to note that CA is not analysing interactions in order to understand what they may represent or "as a pathway to putative mental objects" (Potter, 2012b, p. 123). Instead, it analyses the actions as being situated in a particular sequential, institutional and rhetorical context (Potter, 2012b). CA also finds that "whatever 
happens in conversation happens within some particular, ultimately unique, context" (Sidnell, 2010, p. 6). Every action within a conversation is shaped by the previous interaction, and also provides the context for the rest of the interaction. The potential relevance of the wider context is made available for the analyst through what is "displayed as relevant and consequential to the participants themselves" (Liddicoat, 2007, p. 8). It is this interest and focus on the sequential, rhetorical and participant context which can make CA such a useful method for analysing online interaction.

CA has been used to analyse a wide of variety of relatively mundane, interpersonal interactions (Holt, 2010; Schegloff, 2000), as well as more institutional interactions (Heritage, 2005). CA has allowed us to understand at a very basic level how conversations are built, how turn-taking is organised, how mutual understanding is maintained and how 'trouble' is dealt with. The use of CA for online interaction has a relatively patchy history. A review of the literature by Paulus and colleagues (2016) found that there were a total of 89 articles which used CA to analyse online interaction between 1994 and 2016. When the authors broke this down by publication year (p.3) they found that between 1994 and 2005 there were only 15 articles, whereas between 2006 and 2015 there were 74, suggesting an incremental growth in the latter decade. Studies which have focused on online interaction has focused on a variety of different platforms such as multi-party chat rooms (Benwell \& Stokoe, 2006; Schönfeldt \& Golato, 2003), instant messaging, online forums (Stommel \& Koole, 2010), Twitter (Housley, Webb, Edwards, Procter and Jirotka, 2017), Periscope (Licoppe \& Morel, 2018) and the music sharing service, Soundcloud (Reed, 2017).

One potential reason why there have been relatively few articles which have used CA to analyse online interaction is the potential challenge in adapting a method which has been used predominantly for spoken interaction and applying it to a type of communication which is not only written, but can often use images and videos as well. Giles and colleagues (2015) have noted there are a number of challenges in simply transferring CA findings and ideas wholesale to online interaction. They highlight issues such as the non-linear development of much online interaction, the ways in which the software might impact upon the interaction, and the potential challenges of analysing such massively multi-party and multi-recipient interactions. It is, therefore, important to consider how to manage these potential limitations. Underpinning much of the CA research on online interaction is lan Hutchby's work on affordances (2001; see also Meredith, 2017; Arminen, Licoppe \& Spagnolli, 2016). The concept of affordances puts forward the possibility that features of technology, such as the separation of message construction and sending or the limitation of the length of a message, can be perceived as having a number of potential actions associated with them. However, the features which are relevant are determined by users and how users choose to use them. Therefore, features of technology can both afford and constrain the interactional potential 
(Hutchby, 2001). For example, on Twitter the limitation on the number of characters in a message could be deemed a constraint on users. However, users developed ways to manage this limitation, such as through 'threading' tweets or using acronyms to limit the number of characters used (as users of text messages had done previously). The concept of affordances moves away from the prospect of a technological deterministic approach, and rather allows for an analysis of online interaction which also demonstrates how the interaction orients to particular technological features. On a practical level using the concept of affordances means that analysts examine the interaction itself first and then explore if and how that interaction orients to the relevant technological features of the medium (Meredith, 2017; Arminen et al., 2016). Using the Twitter example, as analysts we would only discuss the limitations on the length of a tweet when users implicitly or explicitly orient to it, such as through using acronyms.

\section{Conversation analysis and online interaction}

To demonstrate how CA works practically when applied to online data, I will start with an example that is taken from an online newspaper comment. The commenter is responding to a news article in which Nigel Farage (the leader of the United Kingdom Independence Party, which campaigned for the UK to leave the European Union), is reported as saying that promises made by the campaign to leave the EU about $\mathrm{f} 350$ million being given to the NHS if the UK left the EU were 'mildly irresponsible'.

\section{Extract 1}

Lol. Can we get some perspective here Mr. Farage? You are the one who led a successful campaign to divorce from the EU. YOU are the one who "declared war" if that's what you call this.

It is firstly important to note how this post can be understood as 'designedly interactional' (Meredith \& Potter, 2013).The post is responding to a newspaper article, and as such the commenter is effectively constructing an adjacency pair between their post and some element of the newspaper article. What is of interest to conversation analysts is how the writer of this message designs it so that it is clearly linked to particular aspects of the article. They use quotation marks around 'declared war' which links to part of the article in which Farage said that "declaration of war" had been issued on any sensible Brexit negotiations. As such, the commenter successfully links their comment back to a specific part of the article - about the Brexit negotiations. Here, then, the commenter designs their post so that it is clearly responsive to the article. 
Extract 1 seems to suggest that it is a response to Nigel Farage. However, it is also a response to the newspaper article itself: in this sense, there are two immediate or direct recipients Farage and the journalist. However, it is also important to note that there is the potential that this post can be responded to by other readers or commenters in the thread, and the post is also designed for them (Meredith, 2016). The post itself could potentially be read as being supportive of Farage, in that successfully leading a campaign or 'declaring war' on the EU could be seen as a positive thing depending on your political views. However, it seems fairly clear that this is actually being critical of Farage, and the commenter uses a number of discursive strategies to indicate their stance. These strategies include the use of 'lol' at the start of the post, which indicates their stance not to their own post - but rather to the content of the article. The commenter also uses capital letters to emphasise the direct recipient of their post, as well as potentially shifting the blame to Farage. The commenter indicates their stance fairly clearly, through a use of traditional discursive strategies which can be used in both spoken and written interaction, as well as stance markers such as 'Iol' which have arisen in the technological era (see also Herring \& Androutsopoulous, 2015; Evans, 2016).

The following extract, which is taken from a Facebook chat (see Meredith, 2017 for a more detailed discussion of this dataset), further shows how the sequential analysis of online interaction can provide additional understanding of online interaction. Isla and Joe are trying to arrange what time to meet at the gym. In the transcript, the time between messages being sent is indicated in brackets and is measured in seconds.

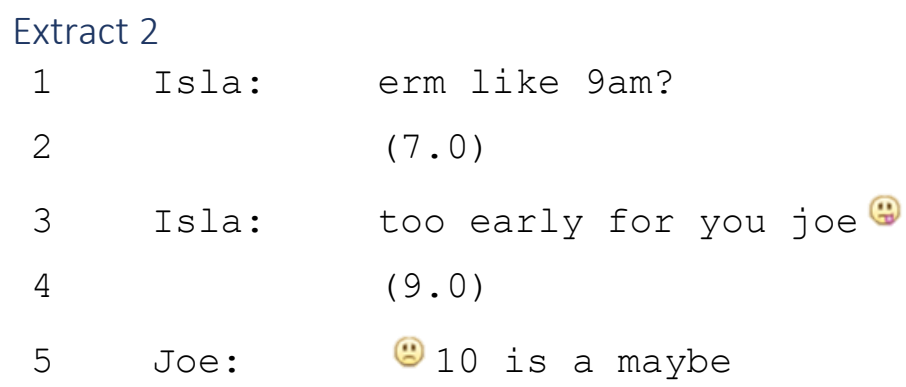

This extract demonstrates how users have found ways of approximating facial expressions to make sure their stance is understood (Markman \& Oshima, 2007). We see both Isla and Joe making use of smilies, with Isla using a face with its tongue sticking out at the end of line 3 , and Joe using a 'sad face' smiley at the start of his turn. From an interactional perspective, what is interesting here is the placement of the smilies. Isla's smiley is at the end of her turn, indicating that the action of her turn is not serious, in the same way as we might understand someone sticking their tongue out in a 
spoken interaction as being non-serious. As such, we can understand her action as something like teasing or joking, rather than, say, asserting or assessing. Joe starts his turn with a smiley, which seems to display his stance towards Isla's turn rather than his own. The action of the smiley may, therefore, differ depending on the context and the sequential position in which it is used. As the features of the internet have developed, we also see emojis, images and gifs (animated pictures) being used to ensure that stance is understood (Tolins \& Samerit, 2016).

Extracts 1 and 2 show how participants have developed resources for indicating stance, and as such, have overcome the reduced cues of the online medium. Such findings are not exclusive to CA, and much research has focused on the resources used for indicating stance (e.g., Derks, Bos, \& von Grumbkow, 2008; Dresner \& Herring, 2010). CA can add to this understanding by demonstrating how these different resources are used within their sequential context, and as designed for particular recipients.

Another issue for theories of online communication is the role that different contexts and relationships plays in interaction. The following example of a Facebook chat opening demonstrates how the relevance of relationship context and technological context can be explored using CA.

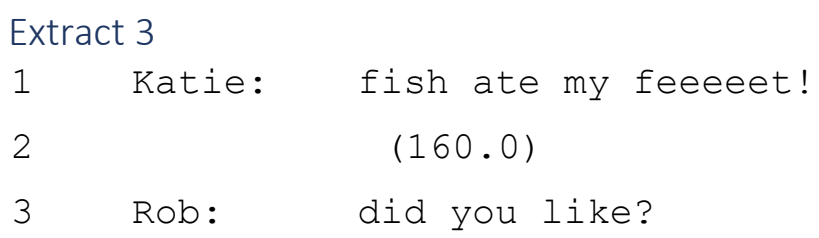

Katie starts her turn with an announcement, which also initiates a topic. There are two key aspects to discuss here: firstly, what this opening might tell us about relationships and context in online interaction. One job that gets done in openings is "the constitution or reconstitution of the relationship of the parties for the present occasion" (Schegloff, 1986, p.113). It is noticeable that there are no greetings or initial pleasantries (e.g., how are you), in this opening, which we might expect at the start of a conversation (Schegloff, 1986). It might seem that this is in some way rude, or lacking in some sort of politeness or facework. However, when we analyse the interaction we can see that Katie's initial turn is not made accountable by the recipient; it is treated as unproblematic. Rob's response also displays an understanding of the topic-initiation, by asking if she enjoyed it (rather than, say, asking for more information, or if it hurt). The response, therefore, suggests some prior knowledge about this topic, so no further elaboration is required. Just from this small fragment of data, we can see that Rob and Katie are close enough that the lack of initial inquires is not an issue which needs to be raised. In addition, we can see that the pair have had some previous communication about Katie's beauty treatment of 'fish eating her feet'. In this sense, then, even 
from a small fragment of data, CA can say something about the relationships between two parties.

The second aspect to examine is how this opening might provide an implicit orientation to the affordances of the medium. It was noted above that there is no greeting sequence, nor is there what has been termed an 'identification and recognition' sequence (Schegloff, 1986); that is, a sequence where the parties ensure they are speaking to their intended interlocutor. We can argue that the omission of these sequences orients to particular affordances of the medium. The names of participants are available on screen and there is also an expectation that, unlike in landline telephone interaction which is the basis of much CA knowledge about openings, the person who owns the Facebook page will be the person who responds. Therefore, there is no need for an identification and recognition sequence. The omission of greetings is, as has already been noted, an indication of the relationship between participants and it is certainly not always the case that greetings are omitted (see Meredith, 2017). However, the lack of greetings can also indicate the potential impact of the medium on the interactional structure. In spoken interaction a greeting token (such as 'hello') can be used as a 'summons', which is a way for indicating that you wish to start an interaction with someone (Schegloff, 1968) as well as doing a greeting. In the online setting, the 'summons' is produced electronically once a message has been sent, and the notification indicates to the recipient that someone wishes to start an interaction with them and that there is a message waiting for them. The message itself can be a greeting, a topic-initiation, a request, a question and so on. Due to the affordances of the medium, the message remains on screen and available to the recipient, so they can read it at any time once they return to their computer. In this way, interlocutors do not need to 'summon' each other immediately because their message will be available. It is in these senses, then, that the opening of this interaction orients not only to the relationship between the participants, but also allows us to analyse the affordances of the medium.

Theories of computer-mediated communication, including social information processing and theory and the hyperpersonal theory, have indicated that the ability to write and re-write messages before they are sent can impact upon the impressions which others form of them. There is concern that as this is a common feature of much online interaction, individuals are becoming uncomfortable having spoken conversations where this is not available to them (Turkle, 2017). However, there is little evidence of what users actually do when they are constructing their messages, and whether they orient to impression management. Data from a screen-captured Facebook chat is shown below, which allows the writing and re-writing of a message to be seen and analysed. Prior to this example, Joe has informed Isla that he has been getting texts from a girl he met the previous night whilst drunk. 


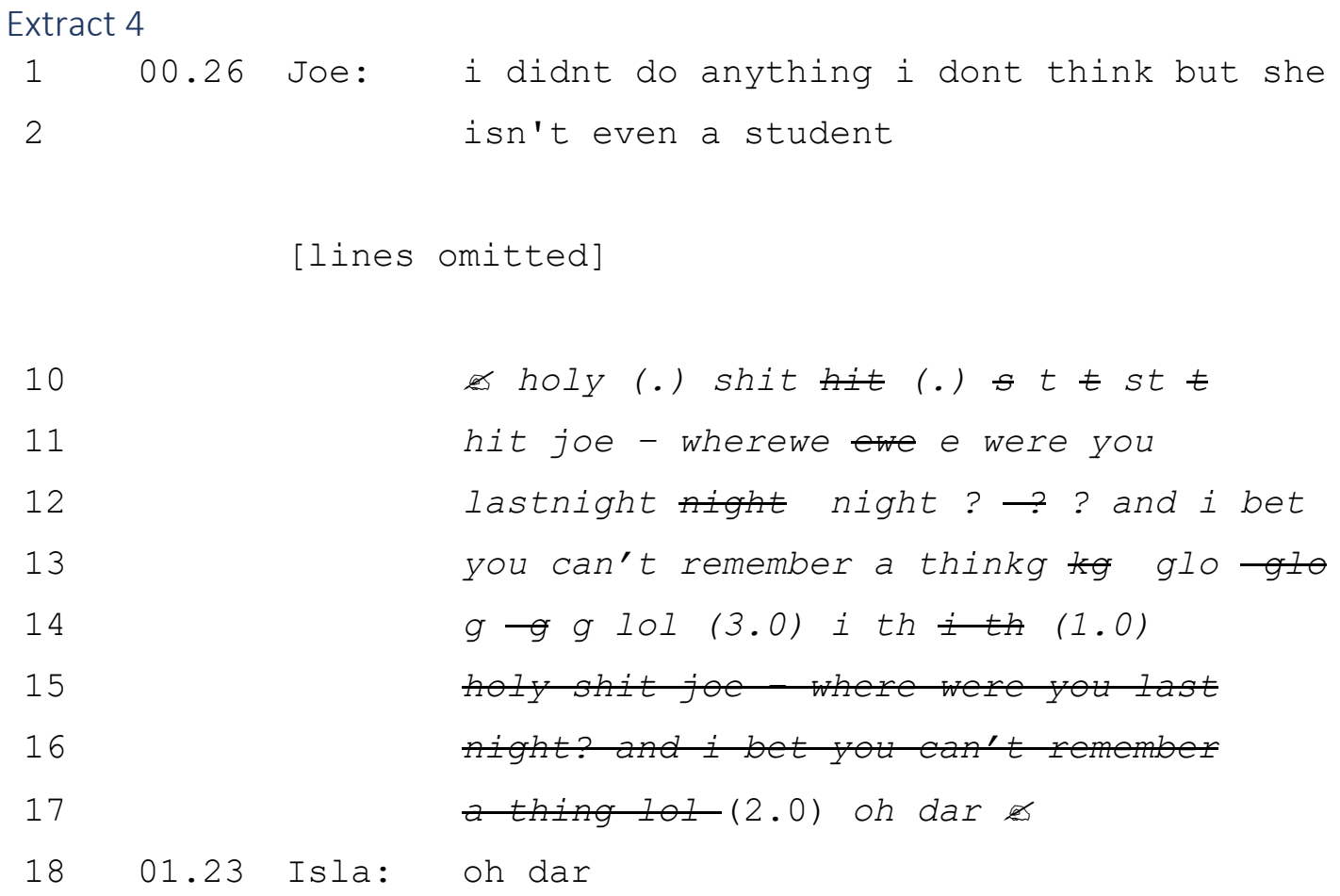

Isla constructs a response to Joe in lines 10-14, which she then deletes before sending "oh dar" at line 18 (which is subsequently corrected to "oh dear"). To understand the consequences of this deletion we can compare the first, aborted attempt, with what is subsequently sent (Drew, Walker \& Ogden, 2013). The original message comprised an inquiry "where were you last night" and an assertion "I bet you can't remember a thing lol", both of which project a response from Joe which would involve a further telling about his evening. Isla completely deletes this and then sends a message which provides some sort of assessment of the evening. In sending a turn which is merely an assessment rather than asking further questions, Isla does not align herself as an interested recipient (Jefferson, 1978) and does not project further talk on the topic of Joe's night out. In other words, while she does not close down the topic (by, say, changing topic or a non-response), neither does she invite further talk on it.

It is possible to make the argument that this post indicates how Isla is managing impressions or face, and indeed others have argued this about message construction (see Ditchfield, 2018). However, from a CA perspective, we can examine how this deletion might orient to the sequential implications of doing this turn, in this place and the potential implications for the subsequent interaction. From this perspective this is not merely about managing impression formation, but rather about managing the direction the conversation may now take. There are clearly differences between doing this when the deletion is 'hidden' and doing it when your interlocutor can hear your deletions. However, by analysing in detail what occurs during message construction we can understand the extent to which what occurs during message editing differs to what is known as 
'repair' in spoken interaction (Meredith \& Stokoe, 2014).

\section{Conclusion}

The development of a myriad of online communicative platforms has led to ever greater interaction online. There has been a concern within social psychology to explore how online, text-based interaction differs from spoken interaction, and a number of theories developed to address this. In this paper, I have argued that rather than necessarily focusing on developing theories which explicate potential reasons for differences between online and offline interaction, we initially need to examine online interaction in depth and 'in the wild' to understand how people interact and how they manage the various affordances of the internet platforms. When we do this, we can see how users orient to particular affordances of the medium in specific contexts, and how they manage the potential constraints of the medium. We can also see how they use the internet to develop and maintain relationships, and can see how particular features - such as hidden message construction can allow for more control over the sequential development of the interaction.

$\mathrm{CA}$ is not necessarily concerned with developing theories of communication, but rather on establishing an evidence-base of real-life interactional practices to understand the ways in which individuals manage their interactions in a variety of contexts. Therefore, CA should be used in an inductive way to allow for online interaction to be analysed on its own terms. One particular benefit of using CA is that there is already a strong evidence base about spoken interactional practices, to which we can compare online interaction. In this way, we can provide empirical evidence of the specific differences between spoken and text-based interaction. There is broad scope for what CA can contribute to the field of social and cyber psychology, including around impression management (Ditchfield, 2018), online dating (Licoppe, Riviere, \& Morel, 2016), online support (Stommel \& Koole, 2010), counselling (Ekberg, Shaw, Kessler, Malpass, \& Barnes, 2016), and cybercrime (Kloess et al, 2015). In analysing a variety of online interactions, CA can offer the potential for an in-depth understanding of how people interact online, and the ways in which this differs based on context and platform, as well as from spoken interaction. As the internet develops, it can also offer the potential for a more longitudinal approach to how online interaction has developed. It is important, though, to be aware of the limitations of applying CA to online interaction. There is a large portion of online communication which is now based on images, gifs, emojis, hyperlinks and so on. While CA may have something to say about the sequential organisation of those types of communication, there are also aspects of the online world, such as liking and sharing posts, which are not commensurate with a CA analysis. As such CA can be used alongside other methods used within social psychology to provide a well-rounded understanding of online behaviour. CA can, though, 
provide a grounded, in-depth approach to exploring and understanding the sequential, rhetorical and contextual elements of online interaction. 


\section{References}

Androutsopoulos, J., \& Beißwenger, M. (2008). Introduction: Data and methods in computermediated discourse analysis. Language@ internet, 5(2).

Antaki, C. (1994). Explaining and arguing: The social organization of accounts. London: Sage.

Antaki, C., \& Widdicombe, S. (Eds.). (1998). Identities in talk. Sage.

Arminen, I., Licoppe, C., \& Spagnolli, A. (2016). Respecifying mediated interaction. Research on Language and Social Interaction, 49(4), 290-309.

Bailey, J., Steeves, V., Burkell, J., \& Regan, P. (2013). Negotiating With Gender Stereotypes on Social Networking Sites: From "Bicycle Face" to Facebook. Journal of Communication Inquiry, 37(2), 91-112. https://doi.org/10.1177/0196859912473777

Benwell, B., \& Stokoe, E. (2006). Discourse and identity. Edinburgh: Edinburgh University Press.

Clayman, S. E. (2013). Conversation Analysis in the News Interview. In J. Sidnell \& T. Stivers (eds). The handbook of conversation analysis. (pp.630-656). Wiley Blackwell.

Cox, J., Nguyen, T., Thorpe, A., Ishizaka, A., Chakhar, S., \& Meech, L. (2018). Being seen to care: The relationship between self-presentation and contributions to online pro-social crowdfunding campaigns. Computers in Human Behavior, 83, 45-55.

Culnan, M. J., \& Markus, M. L. (1987). Information technologies. In F. M. Jablin, L. L. Putnam, K. H. Roberts, \& L. W. Porter (Eds.), Handbook of organizational communication: An interdisciplinary perspective (pp. 420-443). Sage Publications.

Daft, R. L., \& Lengel, R. H. (1986). Organizational information requirements, media richness and structural design. Management science, 32(5), 554-571.

Derks, D., Bos, A. E., \& Von Grumbkow, J. (2008). Emoticons in computer-mediated communication: Social motives and social context. Cyberpsychology \& behavior, 11(1), 99-101.

Ditchfield, H. (2018). Behind the screens of Facebook: an interactional study of pre-post editing and multicommunication in online social interaction (Doctoral dissertation, Department of Media and Communication).

Dresner, E., \& Herring, S. C. (2010). Functions of the nonverbal in CMC: Emoticons and illocutionary force. Communication theory, 20(3), 249-268.

Drew, P., Walker, T., \& Ogden, R. (2013). Self-repair and action construction. In M. Hayashi, G. Raymond \& J. Sidnell (Eds.), Conversational repair and human understanding (pp. 71-94). Cambridge: Cambridge University Press.

Edwards, D. (2004). Discursive psychology. In K. L. Fitch, \& R. E. Sanders (Eds.), Handbook of language and social interaction (pp. 257-273). Mahwah, NJ: Lawrence Erlbaum 
Ekberg, S., Shaw, A. R., Kessler, D. S., Malpass, A., \& Barnes, R. K. (2016). Orienting to emotion in computer-mediated cognitive behavioral therapy. Research on Language and Social Interaction, 49(4), 310-324.

Ellison, N., Heino, R., \& Gibbs, J. (2006). Managing impressions online: Self-presentation processes in the online dating environment. Journal of computer-mediated communication, 11(2), 415441.

Evans, A. (2016). Stance and identity in Twitter hashtags. Language@ internet, 13(1).

Farrer, J., \& Gavin, J. (2009). Online dating in Japan: A test of social information processing theory. CyberPsychology \& Behavior, 12(4), 407-412.

Fullwood, C. (2015). The role of personality in online self-presentation. In A. Attrill (ed). Cyberpsychology. (pp.9-28) Oxford University Press.

Giles, D., Stommel, W., Paulus, T., Lester, J., \& Reed, D. (2015). Microanalysis of online data: The methodological development of "digital CA". Discourse, Context \& Media, 7, 45-51.

Goodwin, C., \& Heritage, J. (1990). Conversation analysis. Annual Review of Anthropology, 19, 283307.

Heritage, J. (2005). Conversation analysis and institutional talk. In K. L. Fitch, \& R. E. Sanders (Eds.), Handbook of language and social interaction (pp. 103-147). Mahwah, NJ: Erlbaum.

Herring, S. C. (2004). Computer-mediated discourse analysis: An approach to researching online behavior. In S. A. Barab, R. Kling, \& J. Gray (Eds.), Designing for virtual communities in the service of learning (pp. 338-376). Cambridge/New York: Cambridge University Press.

Herring, S. C., \& Androutsopoulos, J. (2015). Computer-mediated discourse 2.0. In D.Tannen, H.E. Hamilton, \& D. Schiffrin (eds). The handbook of discourse analysis ( $2^{\text {nd }}$ edition) pp.127-151. Wiley Blackwell.

Holt, E. (2010). The last laugh: Shared laughter and topic termination. Journal of Pragmatics, 42(6), 1513-1525.

Housley, W., Webb, H., Edwards, A., Procter, R., \& Jirotka, M. (2017). Digitizing Sacks? Approaching social media as data. Qualitative Research, 17(6), 627-644.

Hutchby, I. (2001). Conversation and technology. Cambridge: Polity Press.

Jefferson, G. (1978). Sequential aspects of storytelling in conversation. In J. Schenkein (Ed.), Studies in the organization of conversational interaction (pp. 219-248). New York: Academic Press.

Kloess, J. A., Seymour-Smith, S., Hamilton-Giachritsis, C. E., Long, M. L., Shipley, D., \& Beech, A. R. (2017). A qualitative analysis of offenders' modus operandi in sexually exploitative interactions with children online. Sexual Abuse, 29(6), 563-591.

Licoppe, C., \& Morel, J. (2018). Visuality, text and talk, and the systematic organization of interaction 
in Periscope live video streams. Discourse Studies, 1461445618760606.

Licoppe, C., Rivière, C. A., \& Morel, J. (2016). Grindr casual hook-ups as interactional achievements. New Media \& Society, 18(11), 2540-2558.

Liddicoat, A. J. (2007). An introduction to conversation analysis. London: Continuum.

Markman, K. M., \& Oshima, S. (2007). Pragmatic play? Some possible functions of English emoticons and Japanese Kaomoji in computer-mediated discourse. Paper presented at the Association of Internet Researchers Annual Conference 8.0: Let's Play! Vancouver, B.C., Canada. 18 October, 2007. Retrieved from http://umdrive.memphis.edu/kmmrkman/www/AolR8MarkmanOshimaFinalDraft.pdf McKenna, K. Y., \& Bargh, J. A. (2000). Plan 9 from cyberspace: The implications of the Internet for personality and social psychology. Personality and social psychology review, 4(1), 57-75.

Meredith, J. (2017). Analysing technological affordances of online interactions using conversation analysis. Journal of Pragmatics, 115, 42-55.

Meredith, J. (2016). Using discourse and conversation analysis to analyse online data. In D Silverman (ed.) Qualitative Research (4th ed). (pp.261-276). London: Sage Publications.

Meredith, J., \& Potter J. (2013) Conversation analysis and electronic interactions: Methodological, analytic and technical considerations. In H. Lim \& F. Sudweeks, (eds) Innovative methods and technologies for electronic discourse analysis. (pp.370-393). Hershey: IGI Global.

Meredith, J., \& Stokoe, E. (2014). Repair: Comparing Facebook 'chat' with spoken interaction. Discourse \& communication, 8(2), 181-207.

Myddleton, J., \& Attrill, A. (2015). Online relationships. In A. Attrill (ed). Cyberpsychology. (pp.49-71) Oxford University Press.

Paulus, T., Warren, A., \& Lester, J. N. (2016). Applying conversation analysis methods to online talk: A literature review. Discourse, context \& media, 12, 1-10.

Pomerantz, A. M., \& Fehr, B. J. (1997). Conversation analysis: An approach to the study of social action as sense making practices. In T. A. van Dijk (Ed.), Discourse studies: A multidisciplinary introduction, volume 2. Discourse as social interaction (pp. 64-91). London: Sage Publications.

Postmes, T., Spears, R., \& Lea, M. (2000). The formation of group norms in computer-mediated communication. Human communication research, 26(3), 341-371.

Potter, J. (2012a). Re-reading Discourse and Social Psychology: Transforming social psychology. British Journal of Social Psychology, 51(3), 436-455. 
Potter, J. (2012b). Discourse analysis and discursive psychology. In H. Cooper (Ed.), APA handbook of research methods in psychology: Vol2. Quantitative, qualitative, neuropsychological and biological (pp. 111-130). Washington: American Psychological Association.

Potter, J. \& Hepburn, A. (2019). Shaming interrogatives: Admonishments, the social psychology of emotion, and discursive practices of behaviour modification in family mealtimes. British Journal of Social Psychology, https://doi.org/10.1111/bjso.12346

Reed, D. J. (2017). Performance and interaction on Soundcloud: Social remix and the fundamental techniques of conversation. Journal of Pragmatics, 115, 82-98.

Schegloff, E. A. (1968). Sequencing in conversational openings. American Anthropologist, 70, 10751095.

Schegloff, E. A. (1986). The routine as achievement. Human Studies, 9, 111-151.

Schegloff, E. A. (2000). Overlapping talk and the organization of turn-taking for conversation. Language in Society, 29, 1-63.

Schegloff, E. A. (2007). Sequence organization in interaction: A primer in conversation analysis. Cambridge University press.

Schönfeldt, J., \& Golato, A. (2003). Repair in chats: A conversation analytic approach. Research on Language and Social Interaction, 36(3), 241-284.

Short, J., Williams, E., \& Christie, B. (1976). The social psychology of telecommunications. John Wiley \& Sons.

Sidnell, J. (2010). Conversation analysis: An introduction. Chichester, West Sussex; Malden, MA: Wiley-Blackwell.

Sproull, L., \& Kiesler, S. (1986). Reducing social context cues: Electronic mail in organizational communication. Management science, 32(11), 1492-1512.

Stokoe, E. (2009). Doing actions with identity categories: Complaints and denials in neighbour disputes. Text and Talk, 20(1), 75-97.

Stommel, W., \& Koole, T. (2010). The online support group as a community: A micro-analysis of the interaction with a new member. Discourse Studies, 12(3), 357-378.

Suler, J. (2004). The online disinhibition effect. Cyberpsychology \& behavior, 7(3), 321-326.

Tolins, J., \& Samerit, P. (2016). GIFs as embodied enactments in text-mediated conversation. Research on Language and Social Interaction, 49(2), 75-91.

Turkle, S. (2017). Reclaiming conversation: The power of talk in a digital age. Penguin.

Walther, J. B. (1992). Interpersonal effects in computer-mediated interaction: A relational perspective. Communication Research, 19, 52-90. 
Walther, J. B. (1996). Computer-mediated communication: Impersonal, interpersonal, and hyperpersonal interaction. Communication research, 23(1), 3-43.

Walther, J. B. (2011). Theories of computer-mediated communication and interpersonal relations. In M.L. Knapp., \& J.A.Daly (eds). The SAGE handbook of interpersonal communication. (pp.443479).Sage Publications. 